\title{
ANALISIS DINÁMICO DE COSTOS DE OPERACIÓN LOGÍSTICA
}

\author{
Sandra Téllez Vázquez \\ Universidad Politécnica de Guanajuato (UPGTO), México \\ Centro de Innovación Aplicada en Tecnologías Competitivas, A.C. (CIATEC) \\ Miguel Gastón Cedillo Campos \\ Instituto Mexicano del Transporte (IMT), México \\ José Alfredo Jiménez García \\ Instituto Tecnológico de Celaya (ITC), México \\ Roberto Baeza Serrato \\ Universidad de Guanajuato Campus Irapuato-Salamanca.(UG), México
}

\begin{abstract}
RESUMEN
Guanajuato es considerada una de las plataformas logísticas más importantes de México debido a que la mayoría de los cruces interoceánicos atraviesan la entidad. De acuerdo con datos estadísticos de Secretaria de Economía el sector de transporte de carga continuará creciendo anualmente entre 15 y 20\%, y desde Guanajuato se maneja el 74\% de la carga consolidada de la región y $9 \%$ del total nacional. Por esta razón la presente investigación realiza un análisis de la fluidez de carga, tomando como base un caso de estudio aplicado a una de las empresas con mayor movimiento en la región. El estudio se centra en desarrollo de un modelo dinámico para evaluar los factores que afectan el consumo de combustible el cual impacta directamente en los costos logísticos de la organización. Para el análisis se consideran las condiciones topográficas de la ruta utilizando como herramienta el software Globalmap, las políticas establecidas en la NOM-012 de pesos y dimensiones de la Secretaria de Comunicaciones y Transportes (SCT), y el tipo de camión a utilizar. El resultado del modelo evalúa factores que pueden variar según la ruta elegida, y permite a la empresa tomar decisiones para mejorar la fluidez de carga dentro de lo establecido en el marco regulatorio de la Secretaría de Comunicaciones y Transportes de México.
\end{abstract}

\section{Introducción}

Un sistema de transporte eficiente requiere de un proceso continuo de planeación y de ajustes conforme la economía y la sociedad cambian en el tiempo (Gradilla-Hernández, 2012). Sin embargo, la industria del transporte a menudo se asocia con muchos problemas que afectan a la economía, la sociedad y el medio ambiente (Rafaa M'raihi, 2015). Si analizamos los factores que intervienen en el sistema de transporte de carga, puede ser de interés el tipo de producto movido, el vehículo empleado, los requerimientos de entrega, o las maniobras de carga y descarga implícitas. Adicionalmente, el transporte inevitablemente genera impactos 
no deseados, como el congestionamiento; los accidentes; la contaminación ambiental; el ruido, y el deterioro de la infraestructura (Moreno-Quintero, 2006).

(Kamakate \& Schipper, 2009) Estudiaron el uso de la energía del transporte de mercancías por carretera en Australia, Francia, Japón y el Reino Unido entre 1973 y 2005, mencionan que se lograron reducciones en el consumo de energía de transporte por carretera no sólo a través de la economía de combustible de los vehículos, sino también con una mejora en la logística y la conducción. Estudios sobre energía han abordado el tema del transporte de mercancías, la mayoría de estas investigaciones han demostrado que la intensidad energética del transporte es mucho mayor que los de otros modos (ferrocarril y agua) para la mayoría de los países (Rafaa M'raihi, 2015). (Vanek \& Campbell, 1999) Exploran las tendencias de consumo e intensidad energética del transporte por carretera para 14 grupos de productos básicos entre 1985 y 1995 en el Reino Unido. Señalan el crecimiento en longitud y complejidad de la oferta de estas cadenas como el principal motor del aumento del consumo de energía de carga y también identificar algunos de los factores determinantes de la intensidad energética tales como la mezcla de vehículos usados y de carga útil promedio. (Sorrella et all, 2009, 2012) estiman la contribución relativa de los diez principales indicadores además del PIB al cambio en el Reino Unido de mercancías por carretera entre el uso de energía 1989 y 2004. Ellos descubren una disociación relativa pero no absoluta del consumo de energía en carretera del transporte de carga del PIB, que se explica principalmente por la disminución del valor de los bienes fabricados en relación al PIB, y también por las reducciones en el peso medio de la carga útil, la cantidad de circular vacío y el uso de combustible por vehículo-kilómetro. (Pérez-Martínez, 2010) Investiga el consumo de energía del transporte de mercancías y las emisiones relacionadas para el período 1990-2007 y las proyecciones para el año 2025. Se determina que el aumento en el crecimiento del uso de energía no se detendrá a menos que hubiera reducciones significativas en las intensidades energéticas de mercancías por carretera, un cambio en el reparto modal y una mejora en el rendimiento promedio de los vehículos de diesel en carretera. Mientras la mayoría de los estudios se enfoca en la literatura mencionada en el consumo de energía, (Lidia Andrés, 2015) centran su investigación en profundizar la comprensión de uno de sus componentes principales, la intensidad energética, variable que es clave en lograr un uso más eficiente de la energía en el transporte. Estos factores son los verdaderos índices de intensidad energética (medida como el consumo de energía por toneladas/kilómetros transportado) y el índice estructural (el relativo cambio en la composición de los transportes de mercancías por carretera). Una forma de abordar el modelado del transporte de carga que ha resultado de utilidad en el ambiente de ingeniería, es el enfoque sistémico, esto debido a que los sistemas de transporte son considerados sistemas complejos por la cantidad de variables y factores que intervienen en su desempeño y funcionamiento.

La dinámica de sistemas (SD) por sus siglas en inglés, es una metodología mediante la cual es posible crear modelos de sistemas con cierto grado de complejidad que interactúan en forma constante con el medio, busca entender el comportamiento y la evolución de estos a través del tiempo (Sterman, 2000). Dentro de este contexto lo mencionado por (CedilloCampos M. G., Sánchez-Ramírez, Vadali, Villa, \& Menezes, 2014) y (Bueno Solano \& Cedillo Campos, 2014) está relacionado con el transporte de encarga, ya que uno de los factores que también afecta el consumo de combustible son las condiciones de operación de las unidades y en estos dos casos los tiempos de espera. Asi mismo otro aspecto estudiado 
bajo un enfoque de dinámica de sistemas es el propuesto por (Hang \& Li, 2010) desarrollan un marco metodológico para evaluar la regulación del peso de camiones en la provincia de Anhui, China, utilizando dinámica de sistemas, se muestra como los autores toman en cuenta todas las variables cualitativas, las traducen a cuantitativas y desarrollan un modelo de simulación para evaluar la gestión de las políticas de vehículos pesados de transporte. (Lyneis, 2000) Recomienda el uso de los modelos de dinámica de sistemas para pronosticar el comportamiento de los mercados, también afirmó que los modelos SD se pueden utilizar no sólo para la toma de decisiones, también para la identificación de las variables en un sistema que tienen el mayor impacto en la toma de decisiones y merecen la mayor atención en el tiempo. La investigación que se presenta en este artículo es un sistema complejo dada la cantidad de variables que intervienen en su elaboración por lo que se utilizará como metodología SD, donde nos enfocaremos a analizar las variables que influyen en el consumo de combustible de una empresa de autotransportes especializada en materiales peligrosos, de la misma manera se adapta un modelo experimental para medir el efecto de la carga y la pendiente en rutas de transporte y su impacto en el consumo de combustible, agregando entre otras variables que se identificaron en el caso de estudio.

\section{Antecedentes}

Para Guanajuato resulta de vital importancia estudiar el comportamiento de este sector debido a la llegada de diferentes empresas, sobre todo concentrándose en la región LajaBajío, aun cuando el mayor porcentaje de empresas establecidas son del ramo automotriz, resulta apropiado estudiar el comportamiento de otros sectores, tal es el caso de las empresas transportistas especializadas en manejo de carga peligrosa, debido a que empresas proveedoras no directas de la industria automotriz se han establecido en Guanajuato y cada vez es más la demanda de este tipo de productos. El autotransporte es un elemento esencial e integral de una economía industrializada moderna. De acuerdo con Secretaría de Comunicaciones y Transporte (SCT, 2015) en México, el transporte de carga representa el $55.5 \%$ de movilización de carga respecto a todos los modos de transporte, del cual $85 \%$ es de carga especializada, y $78 \%$ transporta material peligroso. Dentro del estado de Guanajuato 20,984 unidades son de consumo diésel. Para el estudio que se realiza se tomó como referencia a la empresa ALZ ubicada en el estado de Guanajuato, manejan 57 unidades de las cuales 46 son tanques C3S3. El Programa Nacional para el Aprovechamiento Sustentable de la Energía (PRONASE) 2009- 2012 sitúa al autotransporte como el área de oportunidad con mayor potencial de ahorro de energía del país, dentro de las actividades del Plan Anual de Trabajo 2015 se han organizado para atender sectores en lo particular, llevar a cabo acciones que cruzan a los sectores (Programas transversales) y realizar acciones de apoyo y soporte a la propia Comisión Nacional de Uso Eficiente de la Energía (CONUEE). En esta nueva versión del Plan Anual de Trabajo se ha incluido un nuevo programa transversal dedicado a los Sistemas de gestión de la energía que incluye el área de transportes y su objetivo es propiciar el uso eficiente de la energía en el sector transporte, favoreciendo el desarrollo de las mejores prácticas y el impulso a las nuevas tecnologías de los vehículos automotores, el uso de combustibles alternos, así como otros equipos. 


\section{Metodología}

El desarrollo propuesto para esta investigación, es una primera parte experimental con las variables elegidas para determinar el comportamiento del sistema de transporte de una empresa que maneja residuos peligrosos. Se desarrolla la siguiente metodología que permitirá seguir analizando las variables que se vayan encontrando y requiriendo para enriquecer el modelo propuesto. La figura 1, muestra los pasos llevados a cabo para el desarrollo de esta investigación donde se incluyen las herramientas utilizadas en cada uno de ellos, para analizar la conceptualización y solución del problema, cada herramienta proporciona datos interesantes para su análisis y futuras investigaciones.

Se realizó una revisión de literatura tomando como referencia para esta investigación lo establecido por (Posada-Henao \& Gonzalez-Calderon, 2013) autores han establecido una metodología concreta para analizar el consumo de combustible en transporte de carga, encontrando el estudio de (Posada-Henao \& Gonzalez-Calderon, 2013) quien realiza una revisión de los modelos existentes en modelos para la estimación del consumo de combustible en vehículos para transporte por carretera, y (Posada Henao, 2012) de igual manera se hace análisis sobre la cantidad de carga posible en los camiones (para lo cual se revisan las normas legales establecidas en cuanto a pesos máximos permitidos para los vehículos que circulan en las carreteras) y su influencia en el consumo de combustible utilizando diseño de experimentos para estudiar la relación de las variables, generando un modelo de regresión lineal que será base de nuestra propuesta. El vehículo en particular que se analiza es el de la marca Kenworth, la razón por la que se utilizan es debido a su reconocimiento en el mercado y al igual que en la empresa ALZ se utilizan estos vehículos para la prestación del servicio que ofertan en México.

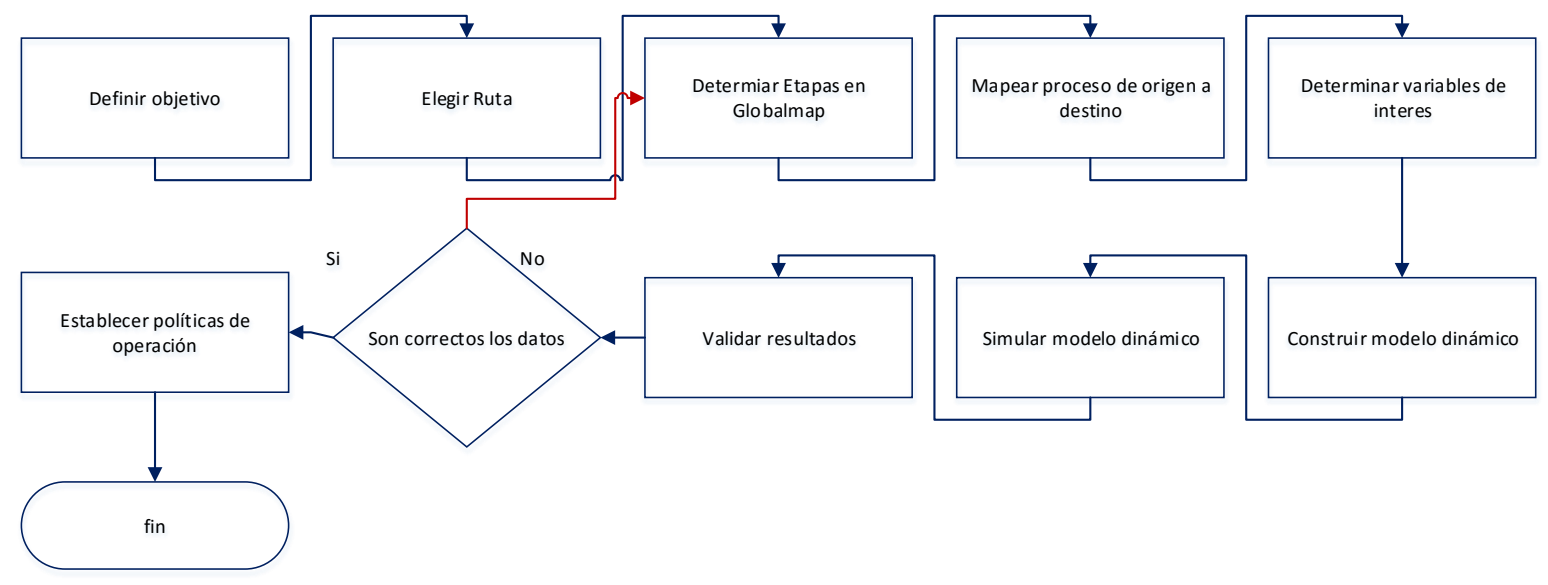

Fig. 1 Metodología Fuente: Elaboración propia

Para el entendimiento del proceso se documentaron diferentes conceptos de las actividades que son pieza clave para el proceso de embarque desde cómo se clasifican las unidades, la 
realización del relleno de diesel, cual es la manera de pago y como se asigna el viaje. Todo ello se representa en un mapeo del proceso que permite visualizar de manera gráfica el comportamiento del sistema estudiado. Así mismo se entrevistaron a cinco de los diez operadores que manejan esa ruta, para tener más claridad en la información de la misma.

Para analizar la ruta carretera se utilizó Globalmap® lo cual ayuda a estudiar las condiciones de la misma respetando las políticas establecidas en la NOM-012 de pesos y dimensiones de la Secretaria de Comunicaciones y Transportes (SCT), se siguen los pasos de SD para definir el comportamiento del sistema. Por todo lo anterior se realizó un mapeo en la cadena de valor de la ruta Altamira-Salamanca y viceversa, incluyendo cada etapa de la altimetría en las pendientes según el comportamiento de la misma, para representarlo se utilizó Value Stream Maping (VSM).

\subsection{Globalmap}

Diversos factores pueden identificarse que provoquen el aumento del consumo de combustible en una unidad de carga, sin embargo pocas empresas tienen establecido la cantidad real consumida de combustible respecto a la cantidad de carga y las condiciones topográficas de una ruta, estas empresas en ocasiones determinan el consumo de combustible en base al rendimiento del motor, y muchas veces sin considerar otros aspectos que afectan el rendimiento del mismo, situación que dentro de una empresa de transporte puede provocar diferencias entre operadores del tracto camión y dueños de la compañía. En este artículo se analizarán dos aspectos importantes relacionados con el consumo de combustible pendiente y mantenimiento, incluyendo variables como velocidad, peso, porcentaje de la pendiente según la inclinación, en el aspecto del mantenimiento y solo para este estudio se incluyen variables de porcentajes de consumo en caso de falta de limpieza de filtros, afinación y presión en llantas. Para medir estas variables se eligió una de las rutas con más bajo rendimiento en el consumo de combustible de la empresa ALZ, la ruta es la que transporta un material toxico del puerto de Altamira Tamaulipas a Salamanca Guanajuato, donde se encuentra ubicado el cliente. La figura 2 muestra la ruta establecida en GlobalMap con los parámetros que se establecieron como tipo de vehículo, velocidades de conducción, tipo de carga y peso, lo que permitirá seleccionar la configuración de su vehículo en base a la NOM012 de pesos y dimensiones de la SCT y con todo ello calcular el trayecto óptimo.

Durante la revisión bibliográfica se encontró que lo propuesto por (Posada Henao, 2012) quien desarrolla dos modelos de regresión lineal con Diseño de Experimentos, en el primero con diseño factorial $3^{\mathrm{k}}$ y en el segundo $2^{\mathrm{k}}$, utiliza en ambos las variables de velocidad, peso y pendiente consideradas para este proyecto y que se definen en el apartado 3.4 de este artículo y en el presente trabajo se le dará el enfoque de Dinámica de Sistemas. Para la investigación y análisis de los datos se llevó a cabo en una sola ruta la cual es Altamira Tamaulipas a Salamanca Gto., en tracto camiones tipo tanque de los cuales se tomaran dos años como muestra 2014. 


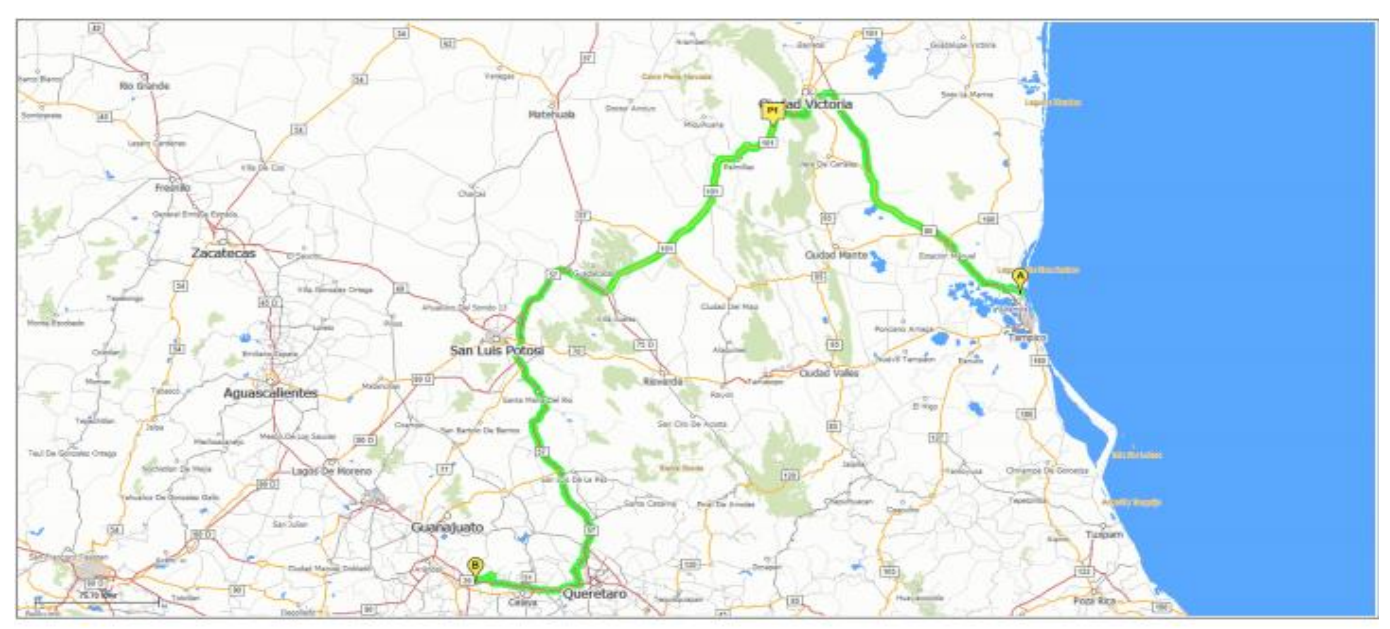

Fig. 2 Ruta Altamira Tamaulipas - Salamanca Guanajuato Fuente: GlobalMap ${ }^{\circledR}$

Para determinar los datos de rellenos, consumos y factores de rendimiento, se extrajeron datos de las unidades que tuvieron viajes en ese periodo. En la figura 3, se muestran los datos topográficos de la ruta que se está estudiando, para lo cual estudiaremos tres tipos de etapas, pendiente ascendente, pendiente descendente y etapa plana.

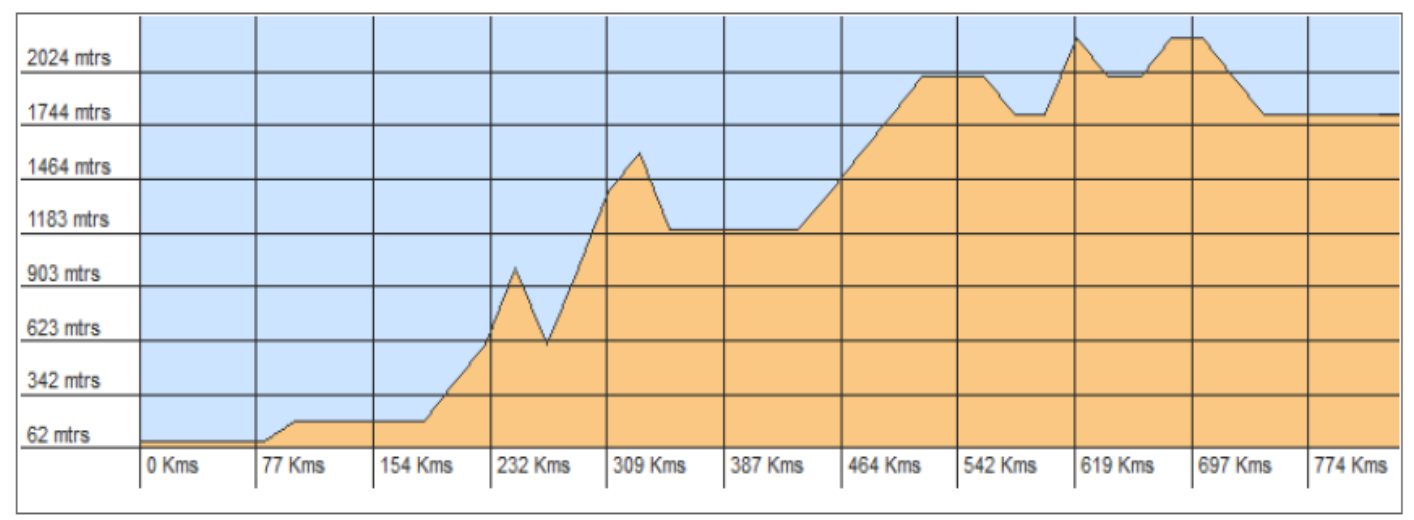

Figura 3. Altimetría de la ruta Altamira Salamanca Fuente: GlobalMap ${ }^{\circledR}$

Los datos que a continuación se describen fueron calculados del software GlobalMap, el software solo muestra el total de kilomentros recorridos y los metros ascendidos en la ruta sobre el nivel del mar, por lo que los siguientes datos donde la ruta marca $823 \mathrm{~km}$ recorridos en 13 horas a una velocidad promedio de $90 \mathrm{~km} / \mathrm{hora}$, determinando tres etapas las cuales son: pendiente ascendente: con un $29.7 \%$ que representa un $244.43 \mathrm{~km}$ recorrido en 3.86 horas, pendiente descendiente: con 14.4\% que representa un $118.51 \mathrm{~km}$ recorridos en 1.9 horas y plano con un 55.9\% que representa un $460.05 \mathrm{~km}$ recorridos en 7.28 horas. Lo que se puede ver en la figura tres es la posición Altamira-Salamanca, ya que es posible ver que se encuentra sobre 62 metros sobre el nivel del mar, esta imagen puede variar si invertimos el origen destino y el porcentaje de la pendiente ascendente se invertiría a ser el porcentaje de la pendiente descendiente. 


\subsection{Mapeo de proceso de la ruta Altamira-Salamanca}

En este apartado se presenta el mapeo de procesos de la ruta Altamira-Salamanca utilizando la herramienta del Value Stream Mapping (VMS), identificando tanto las actividades en cada etapa del proceso y las condiciones topográficas de la misma, información necesaria para agregar en el modelo propuesto. En el VSM el transporte es una de las actividades que no agrega valor en la cadena, pero para una empresa de transportes justamente el transporte es una actividad que agrega valor a todo el proceso, pues es la actividad principal del sistema.

En la figura 4 se muestran las seis etapas que se identificaron y que son importantes a considerar en el consumo de combustible en la ruta, siendo estas primero cuando se dirige por el producto, ya que las unidades se localizan en Celaya Gto., y les informan que tienen que ir por producto al puerto de Altamira, por lo que viajan por el producto hacia el puerto sin carga y solo se considera el peso propio del camión de 19 toneladas, lo que podemos identificar como actividades que no agregan valor al proceso, pues no llevan ningún producto, posteriormente cuando llegan al puerto cargan el producto y regresan cargados trayendo un peso de 33 toneladas promedio, por lo que en el regreso se considera 52 toneladas promedio, como se había mencionado anteriormente, en el modelo se analizará el efecto de la carga en el consumo de combustible y se realizará la propuesta de incluir otras variables que se agregan de acuerdo con la información recolectada.

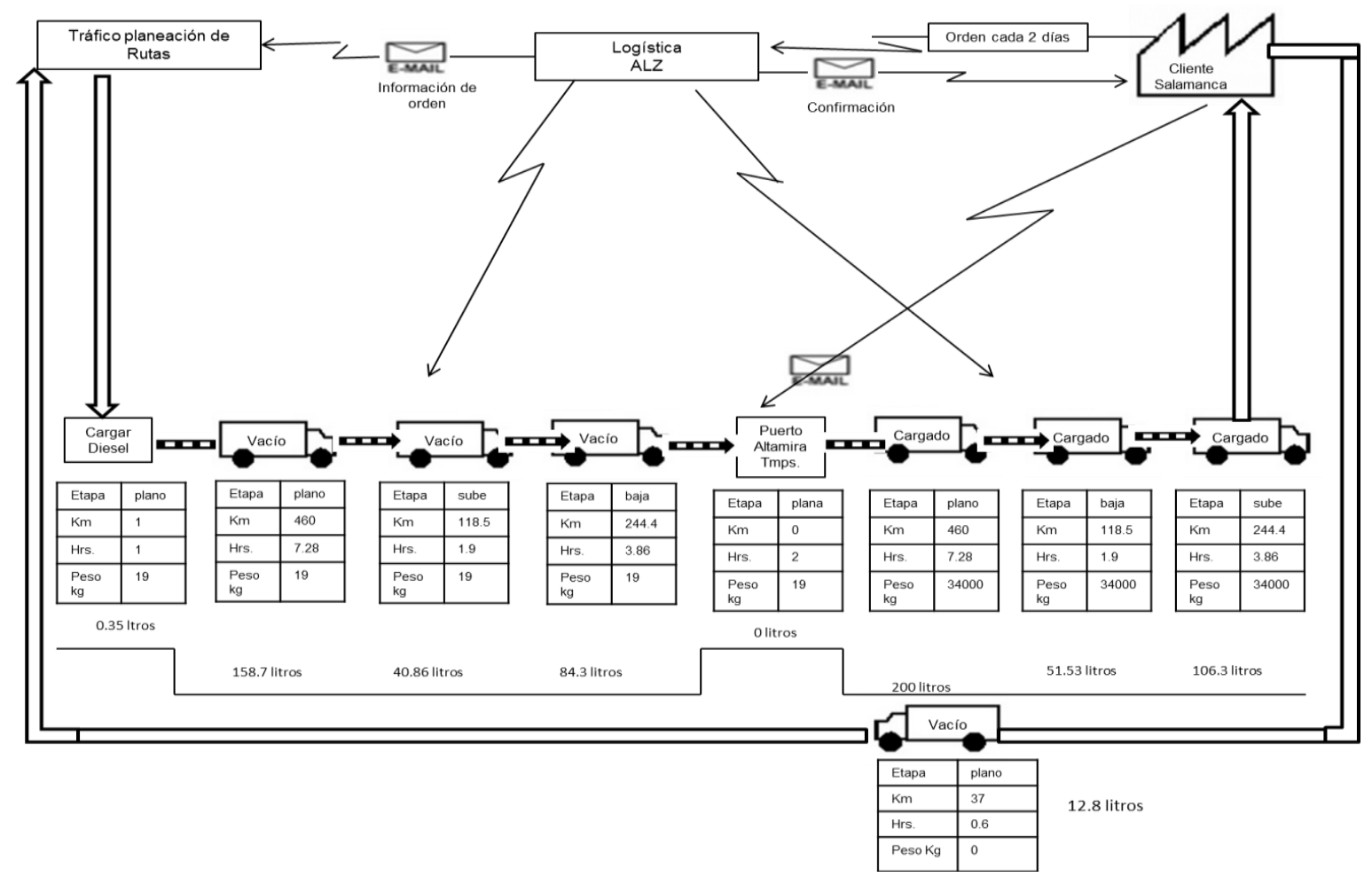

Figura 4.Mapeo de Proceso de las etapas en la ruta Altamira-Salamanca Fuente: Elaboración propia 


\subsection{Estructura del modelo}

Para esta primera experimentación las variables identificadas son algunas de las encontradas en la revisión de la literatura, así como también las proporcionadas por los operadores de la empresa ALZ las cuales están representadas dentro del VSM, siendo estas, las características del vehículo, peso de la carga transportada, topografía de la carretera por la que se transita, velocidad permitida, condiciones de mantenimiento del vehículo, por lo que se considera dentro de la estructura de costos de operación vehicular como una componente variable. (Posada Henao, 2012) Determina el efecto de la cantidad de carga que moviliza un camión, en el consumo de combustible cuando transita en régimen de flujo libre, desarrolla un diseño factorial $3^{\mathrm{k}}$ y $2^{\mathrm{k}}$ los cuales se describen en la tabla 1 .

\begin{tabular}{|l|c|c|c|c|}
\hline \multirow{2}{*}{ Factor } & \multicolumn{2}{|c|}{ Diseño 3 } & \multicolumn{2}{c|}{ Diseño $2^{\mathrm{k}}$} \\
\cline { 2 - 5 } & Niveles & Valores & Niveles & Valores \\
\hline Pendiente carretera (\%) & 3 & $7.0,5,2.0$ & 2 & $2.0,0.6$ \\
Peso total del vehículo(t) & 3 & $51.95,26.28$, & 2 & $51.95,19.09$ \\
Velocidad del & 3 & 19.09 & 2 & 60,40 \\
vehículo(km/h) & & $35,30,25$ & & \\
\hline
\end{tabular}

Tabla 1- Factores, niveles y valores de niveles - camión C3S3

Fuente: (Posada Henao, 2012)

El camión utilizado como prueba en este diseño, tiene las mismas características de motor (Cummins) y año (2007) con el que cuentan en la empresa ALZ, utilizados en la ruta Altamira-Salamanca, por ello se realizará la prueba comparativa entre ambos casos. Con la información anterior el autor obtiene los siguientes modelos de regresión lineal para cada diseño quedando de la siguiente manera:

Para el diseño $3^{\mathrm{k}}$ se generó la ecuación 1

$$
\begin{aligned}
& C=-49.3166 \mathrm{P}+30.2423 \mathrm{~V}+20.6906 \mathrm{P} * \mathrm{p}+0.355453 \mathrm{P}^{2}-24.9639 \mathrm{p}^{2} \\
& -0.514948 \mathrm{~V}^{2}-0.021823 \mathrm{P}^{2}
\end{aligned}
$$

Y para el diseño experimental $2^{\mathrm{k}}$ la ecuación 2 es:

$\mathrm{C}=11.6719 \mathrm{P}-1.79316 \mathrm{~V}+2.9518 \mathrm{P} * \mathrm{p}+0.0931592 \mathrm{P} * \mathrm{~V}-0.136448 \mathrm{P}^{2}$

Dónde:

$\mathrm{C}=$ consumo $(\mathrm{ml} / \mathrm{km})$

$\mathrm{P}=\operatorname{Peso}(\mathrm{t})$

$\mathrm{p}=$ Pendiente $(\%)$

$\mathrm{V}=$ Velocidad $(\mathrm{km} / \mathrm{h})$ 
Otras variables que se agregaron son las relacionadas con el mantenimiento, como son afinación, presión de llantas y filtros sucios, ya que los operadores mencionan que ellos notan que cuando no le dan el mantenimiento adecuado o cuando se requiere a las unidades, el consumo de combustible se incrementa, por lo que las variables anteriores están representadas en el modelo con valores de $30 \%, 5 \%$ y $10 \%$ respectivamente. Esto sucede en varias ocasiones ya que por exceso de trabajo y falta de unidades en la empresa se tiene que posponer el mantenimiento de las mismas en algunas ocasiones.

\subsection{Modelo Dinámico}

Una vez identificadas las variables de interés, seguimos para construir el diagrama causal para comprender las relaciones que influyen en el comportamiento del sistema. En la figura 5 el diagrama causal propuesto se evalúan el primer modelo experimental con diseño factorial $3^{\mathrm{k}}$, propuesto por (Posada Henao, 2012). Cada etapa evaluada en el mapeo de proceso está representada por una variable de flujo, la cual concentra el valor de las variables auxiliares con los valores descritos en el apartado 3.4.

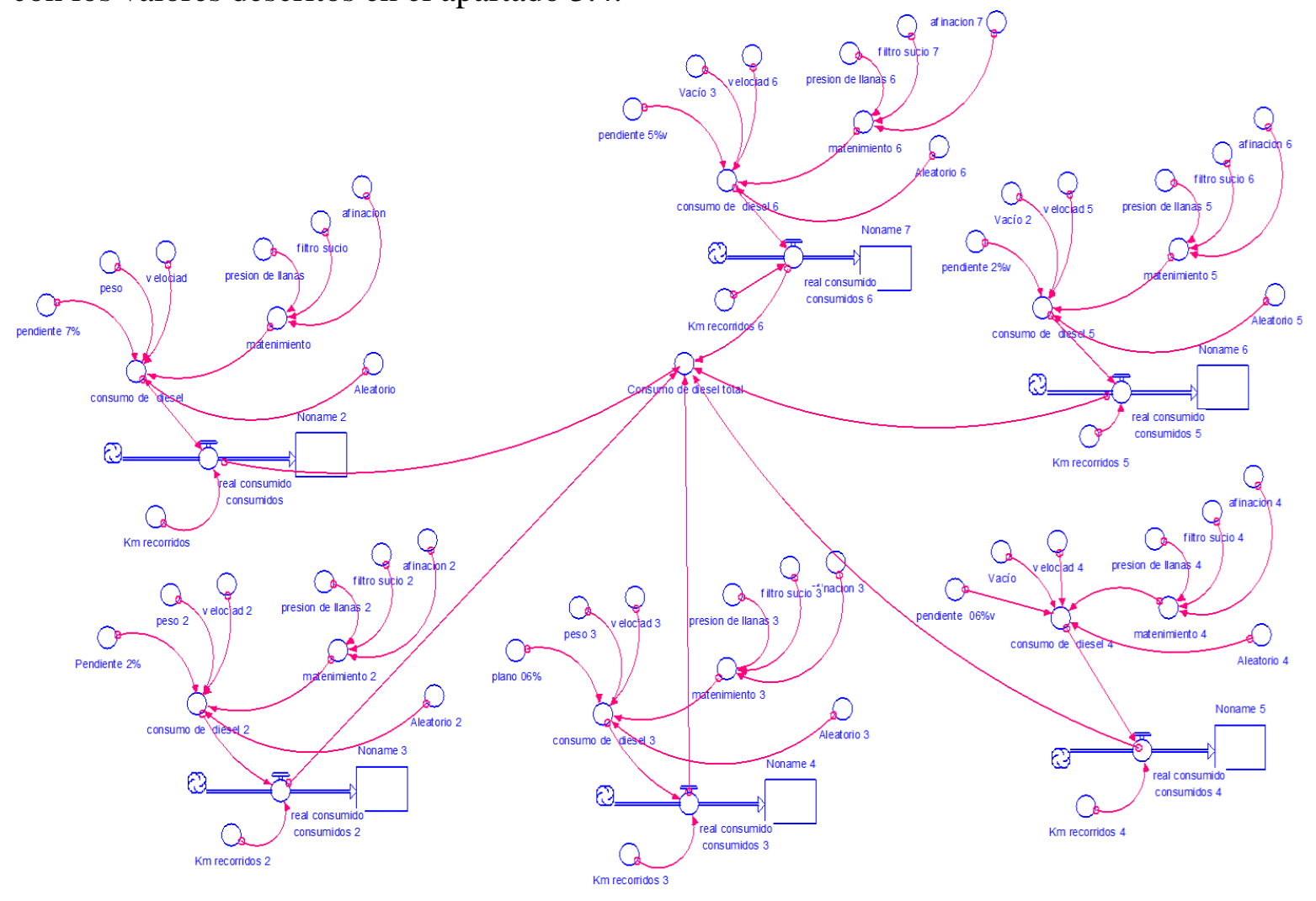

Fig.-5 Diagrama causal 1 con diseño factorial $3^{\mathrm{k}}$ Fuente: elaboración propia

En la figura 6 se muestra el diagrama causal con diseño factorial $2^{\mathrm{k}}$ el cual demuestra un mejor resultado respecto al diagrama causal de la figura 5 que corresponde al diseño factorial $3^{\mathrm{k}}$ y los datos proporcionados por la empresa ALZ. Demostrando con ello lo expresado por 
(Chesher y Harrison, 1987) quienes mencionan que el comportamiento del consumo de combustible tiene una curva en " $U$ " en la cual el mayor consumo de combustible se presenta para velocidades relativamente altas o bajas, donde las velocidades bajas está entre 30 y 40 $\mathrm{km} / \mathrm{hra}$ y velocidades altas más de $80 \mathrm{~km} / \mathrm{hra}$.

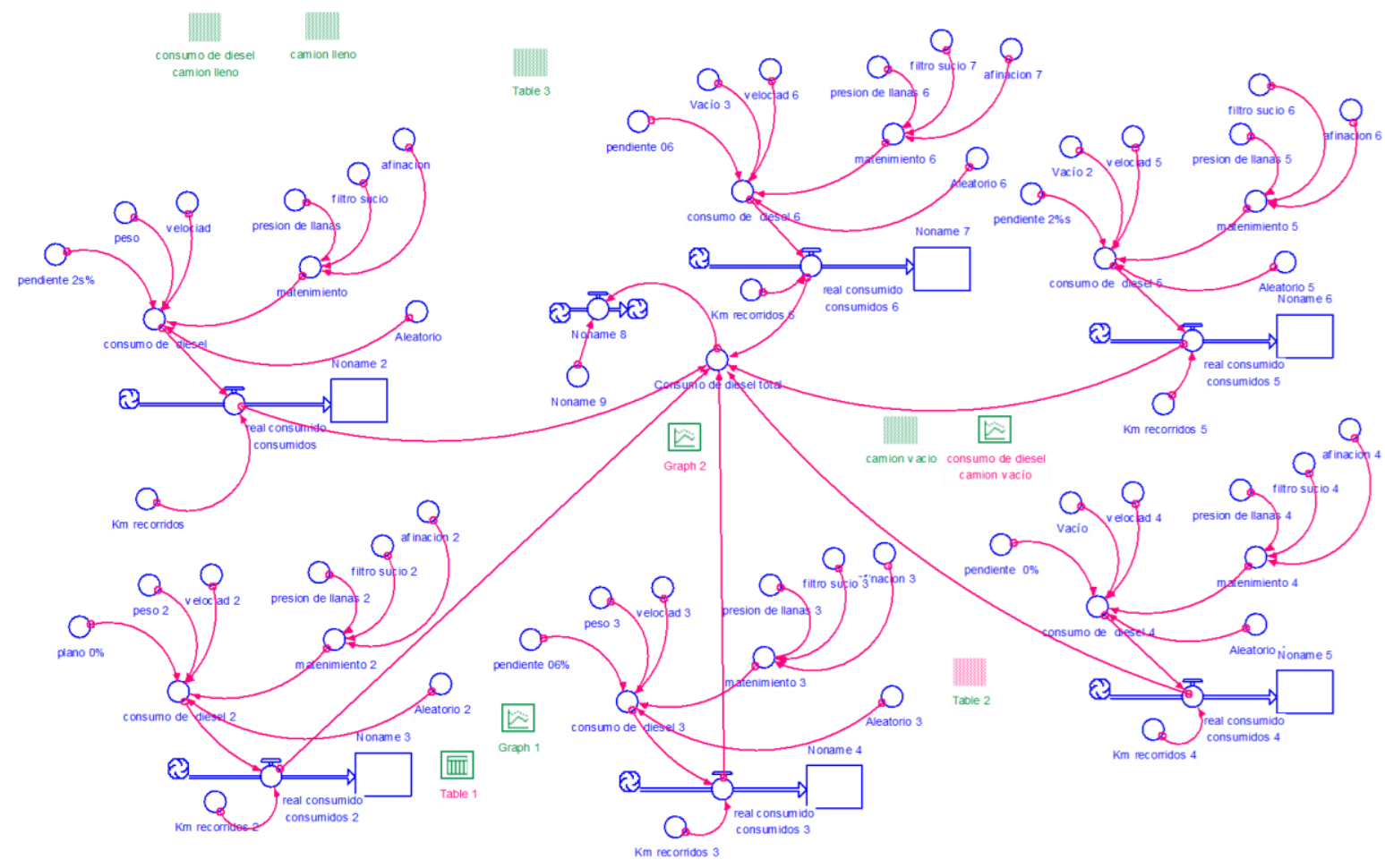

Fig.-6. Diagrama causal 2 con diseño factorial $2^{\mathrm{k}}$ Fuente : Elaboración propia

\subsection{Análisis de datos}

A continuación se muestran los resultados obtenidos por ambos modelos, la validación se dá con el resultado que tiene la propia empresa, comparando el índice de rendimiento y el costo total de combustible que esperan ellos tener. La empresa espera que las unidades alcancen un rendimiento de 2.45 litros por kilómetro, el modelo fue diseñado considerando las variables que la empresa nos ha proporcionado al momento de calcular el índice de rendimiento con el cual miden al operador, de acuerdo con la base de datos proporcionada por la propia empresa lo que se muestra en las figura 7 son los índices que han tenido los operadores. 


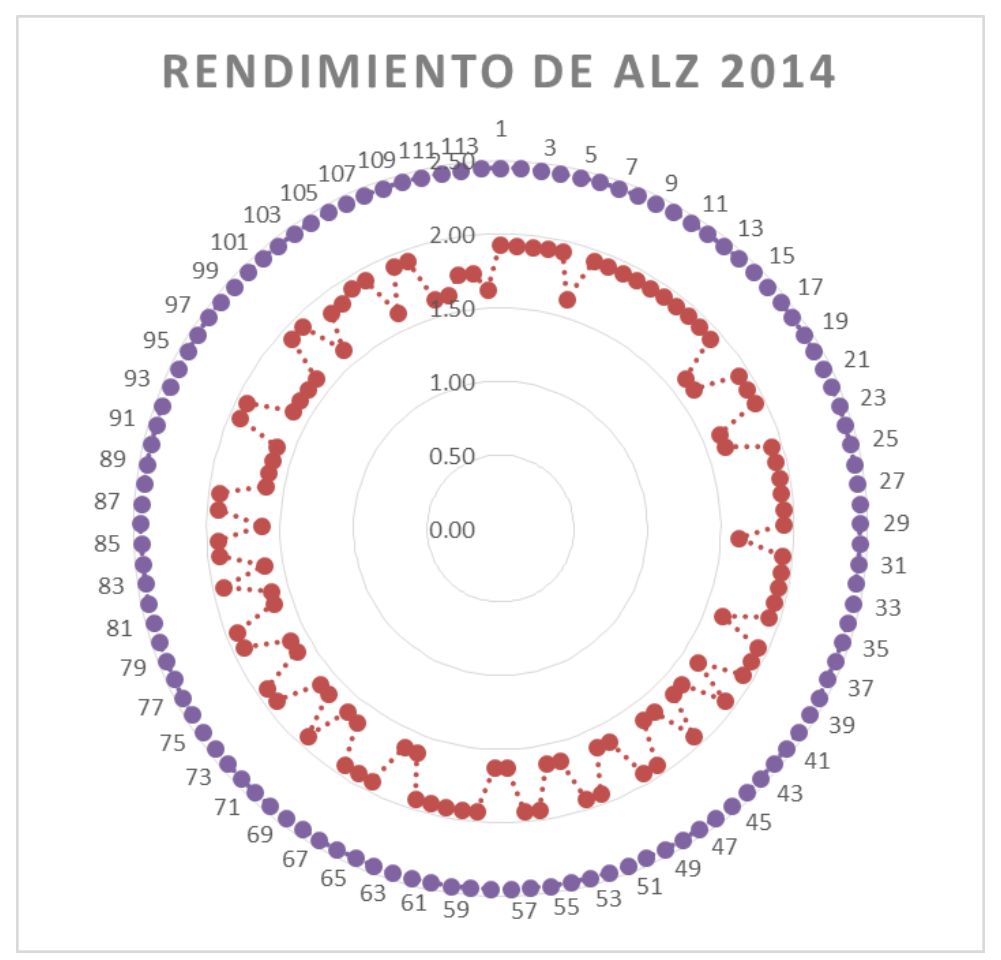

Fig.-7 Rendimiento lt/km en la ruta Altamira-Salamanca

En la figura 7 los puntos purpura muestra el rendimiento que la empresa espera, y los puntos rojos muestra el rendimiento promedio que traen los operadores el cual es de 1.81 , lo que indica que no están alcanzando lo que la empresa espera y esto genera conflicto entre ambas partes debido a que se cree que el operador extrae el combustible, generando altos costos para la empresa debido a que no se alcanza la meta esperada de $2.451 / \mathrm{km}$, lo cual al realizar el diseño con el modelo de simulación se busca comprobar.

\subsubsection{Resultados diagrama causal 1 diseño $3^{\mathrm{k}}$}

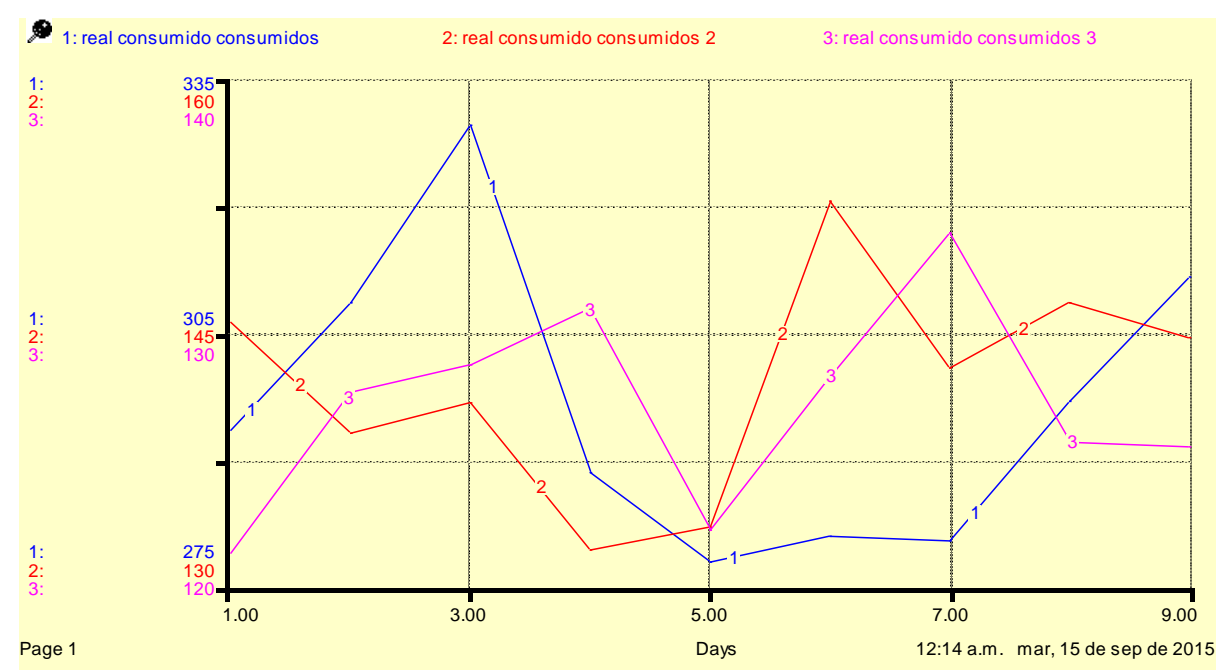

Fig.-8. Consumo de combustible, camión lleno (lt/km)

This work is licensed under a Creative Commons Attribution-NonCommercial-NoDerivatives 4.0 International License (CC BY-NCND 4.0). 


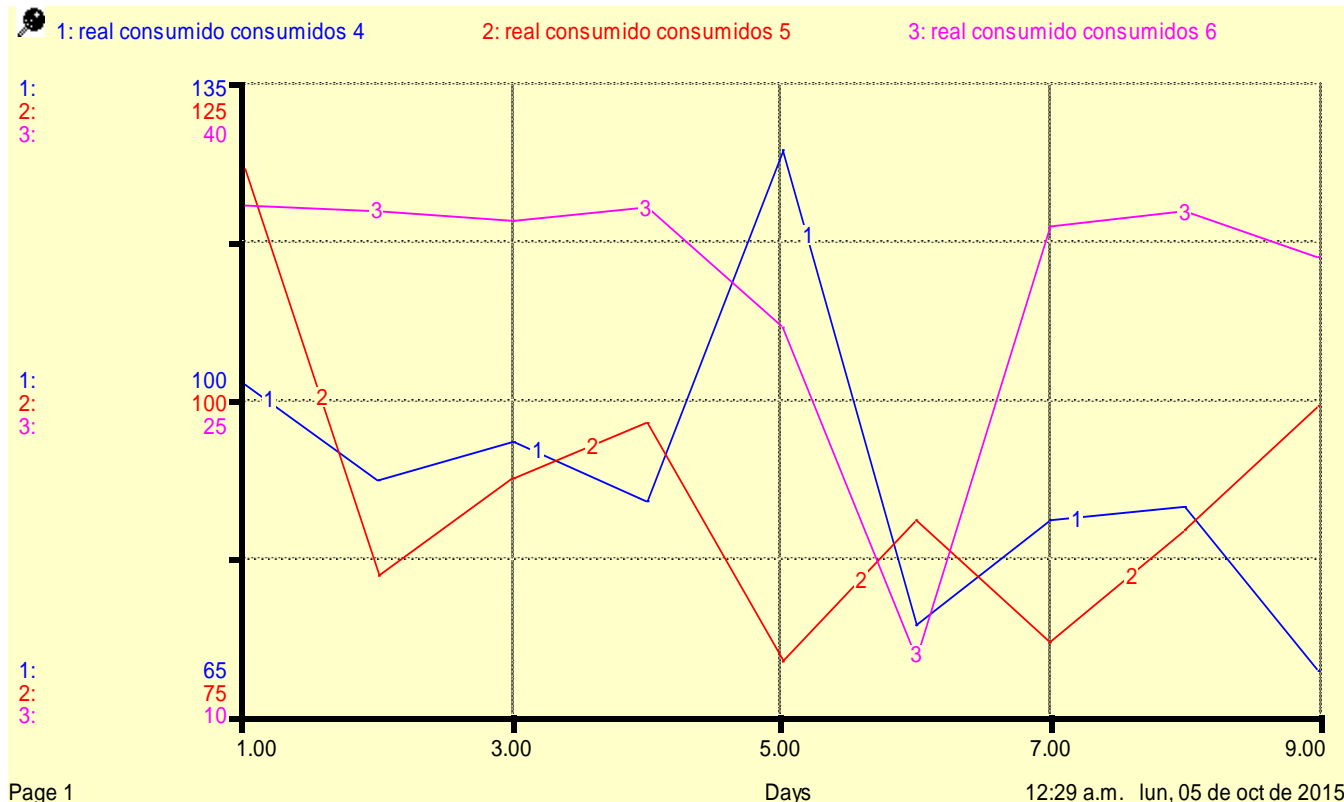

Page 1

Fig.- 9. Consumo de combustible, camión vacío (lt/ $/ \mathrm{km})$

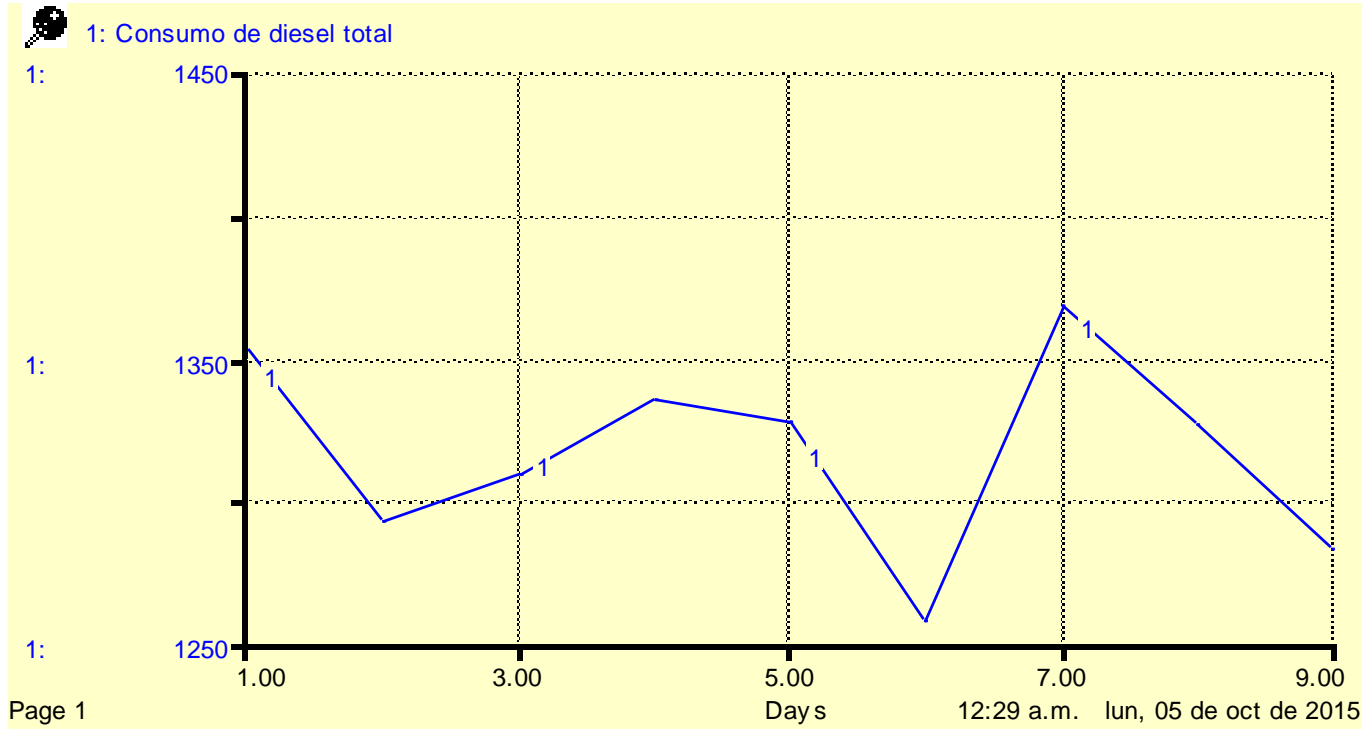

Fig.- 10. Consumo de diesel total $(\mathrm{km} / \mathrm{lt})$ 
1:

1: indice de rendimiento

1:

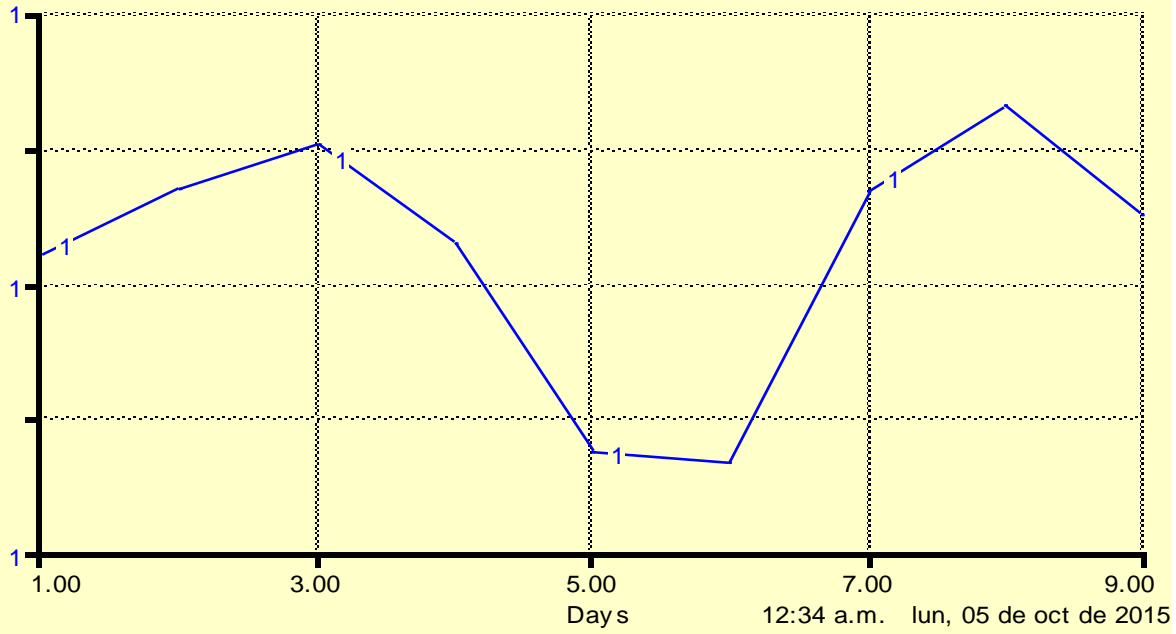

Page

Fig.-11. Índice de rendimiento del diagrama causal 1 con diseño factorial $3^{\mathrm{k}}$

\subsubsection{Resultados diagrama causal 1 diseño $2^{\mathrm{k}}$}

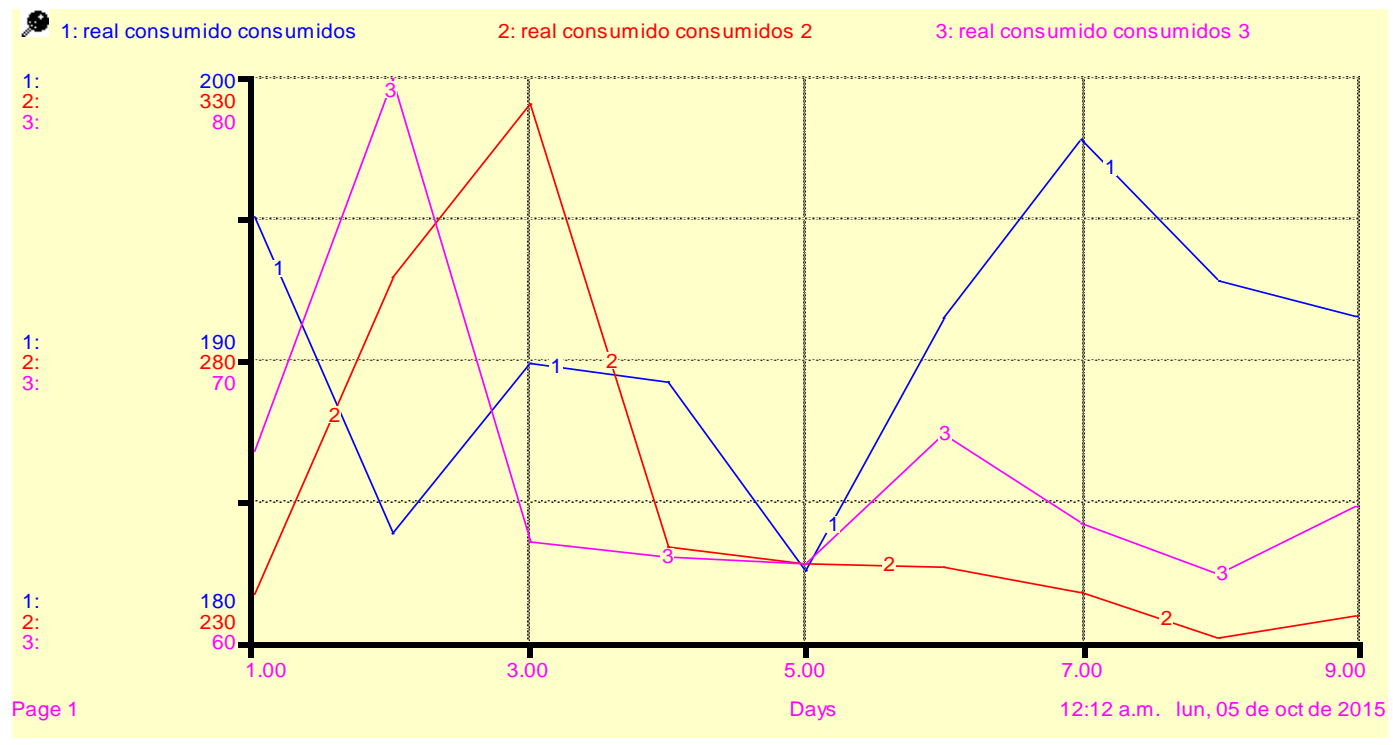

Fig.-12. Consumo de combustible, camión lleno (lt/km) 


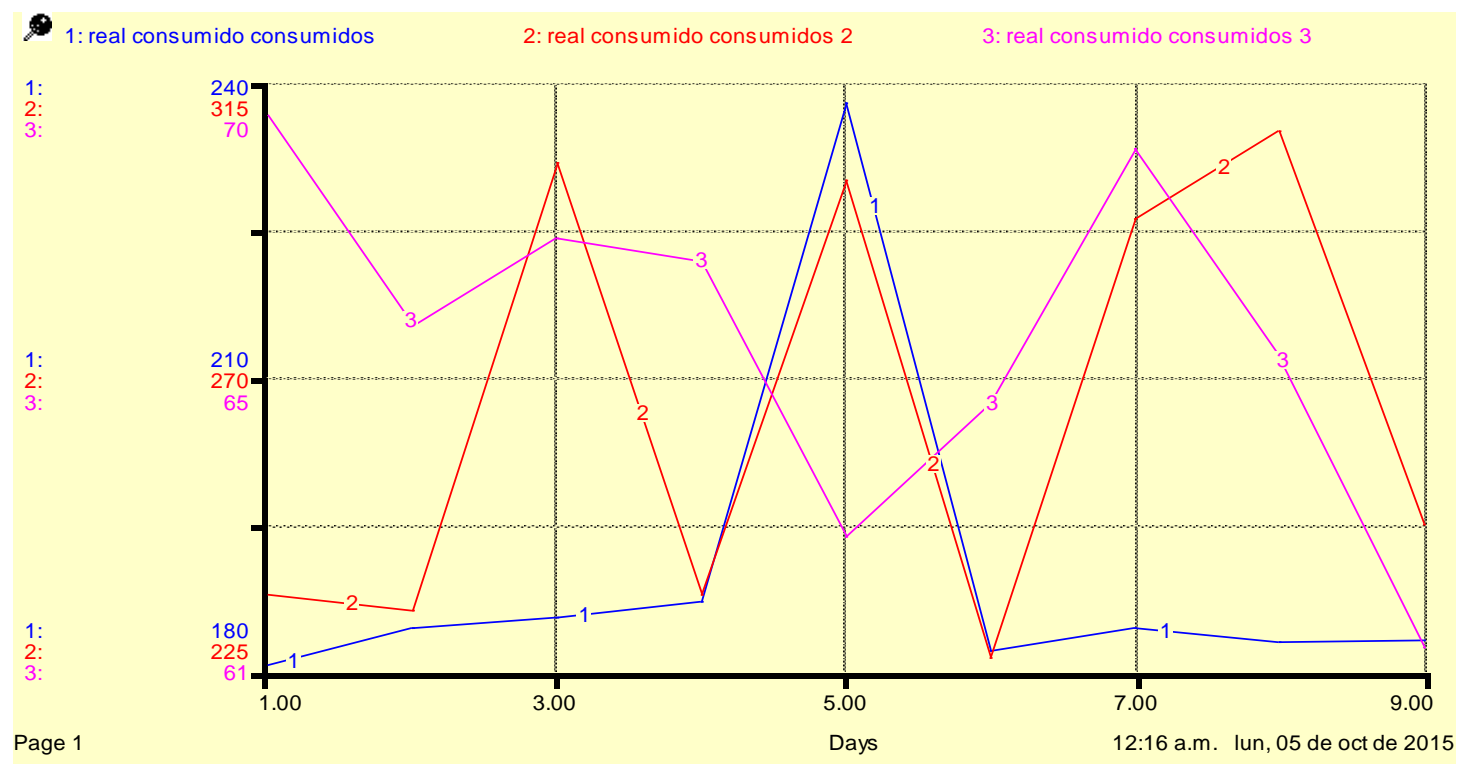

Fig.-13. Consumo de combustible, camión vacío (lt/km)

\section{Conclusiones}

El incremento del peso del vehículo (carga transportada) afecta de manera importante el consumo de combustible, especialmente para pendientes altas y muy particularmente por encima del $5 \%$. De esto se concluye adicionalmente que no es adecuado, por lo menos para carreteras que sean corredores viales para movilización de carga, tener pendientes altas por que hacen que el transporte no sea eficiente debido a los altos consumos de combustible; puede ser preferible una carretera con pendiente baja en lugar de una con pendiente alta aunque su longitud sea mayor ya que seguramente los costos totales de transporte, compuestos básicamente por la inversión y los costos para los usuarios, serán menores, generando así bienestar social.

Podemos concluir de igual manera que el modelo que mas se acerca a la realidad de la empresa ALZ es el segundo, debido a que está cerca del índice de rendimiento establecido por la misma, aún cuando faltan variables que agregar para que sea mas cercano a la realidad. Pues sabemos de antemano que los operadores traen un índice de rendimiento del 2.3 $\mathrm{km} / \mathrm{litro}$. Asi como también podemos concluir que no es posible manejar un factor de rendimiento igual para todos los tipos de rutas debido a que algo que comentan los operadores es que en cada ruta suceden situaciones que la empresa no está dispuesta a considerarles, como accidentes, reparación de carreteras, y eso va disminuyendo el rendimiento del combustible. 


\section{Referencias}

1. Arroyo Osorno, J. A., Aguerrebere Salido, R., \& Torres Vargas, G. (2014). Costos de operación base de los vehiculos representativos del transporte interurbano 2014. Queretaro, México.: Instituto Mexicano del Transporte.

2. Astiz, I. (12 de 02 de 2012). Grafico Radar. Obtenido de Grafico Radar: http://www.fuerzatres.com/2012/02/grafico-radar-html/

3. Bueno Solano, A., \& Cedillo Campos, M. G. (2014). Dynamic impact on global supply chains performance of disruptions propagation produced by terrorist acts. Transportation Research Part E, 1-12.

4. Cedillo-Campos, M. G., Sánchez-Ramírez, C., Vadali, S., Villa, J. C., \& Menezes, M. B. (2014). Supply chain dynamics and the "cross-border effect"': The U.S.Mexican border's case. Computers \& Industrial Engineering, 261-273.

5. Cedillo-Campos, M. G., Sánchez-Ramírez, C., Vadali, S., Villa, J. C., \& Menezes, M. B. (2014). Supply chain dynamics and the "cross-border effect"': The U.S.Mexican border's case. Computer and Industrial Engieneering, 261-273.

6. Chessman de Rueda, S. (13 de 04 de 2011). Conceptos Básicos en Investigación. Recuperado el 03 de 03 de 2015, de Conceptos Básicos en Investigación.

7. CONUEE, C. N. (2015). Plan Anual de Trabajo 2015. México, DF.: Secretaría de Energía.

8. Garcia, J. M. (2003). Teoria y Ejercicios Practicos de Dinamica de Sistemas. En J. M. Garcia, Teoria y Ejercicios Practicos de Dinamica de Sistemas (pág. 300). JMG.

9. Gradilla-Hernández, L. A. (2012). Transporte de carga en México: transición hacia un sistema sustentable. Sanfandilla Queretaro.: Instituto Mexicano del Transporte.

10. Hang, W., \& Li, X. (2010). Application of system dynamics for evaluating truck weight regulations. Transport Policy, 240-250.

11. Hearing, \& Peter. (2000). Historias y Biografias. En P. Herring, Ultimate Train (pág. 148). London. 
12. IDAE Instituto para la Diversificacion y Ahorro de la Energia . (06 de 04 de 2006). Guia para la Gestion del Combustible en las Flotas de Transportes por Carretera. Recuperado el 03 de 06 de 2015, de Guia para la Gestion del Combustible en las Flotas de Transportes por Carretera.

13. Kamakate, F., \& Schipper, L. (2009). Trends in truck freight energy use and carbon emissions in selected OECD countries from 1973 to 2005. Energy Policy, 3743-3751.

14. Lidia Andrés, E. P. (2015). Energy intensity in road freight transport of heavy goods vehicles in Spain. Energy Policy, 309-321.

15. Lyneis, J. M. (2000). System dynamics for market forecasting and structural analysis. System Dynamic Review, 3-25.

16. Moreno-Quintero, E. (2006). Análisis Comparativo de la Modelación del Autotransporte: Carga vs Pasajeros. Sanfandilla, Queretaro: Instituto Mexicano del Transporte.

17. Papacostas, C., \& Prevedouros, P. (1993). Transportation Engineering and Planning. Estados Unidos: Prentice-Hall International.

18. Pérez-Martínez, P. (2010). Freight Transport, Energy Use, and Emission Trends in Spain . Transportation Research Board, 16-22.

19. Posada Henao, J. J. (2012). Efecto de la cantidad de carga en el consumo de combustible en camiones. Medellin, Colombia: Universida Nacional de Colombia.

20. Posada-Henao, J. J., \& Gonzalez-Calderon, C. (2013). Consumo de Combustible en Vehículos para transporte por carretera-Modelos Predictivos-. Revista Ingenierías Universidad de Medellín, 35-46.

21. Rafaa M'raihi, T. M. (2015). Carbon emissions growth and road freight: Analysis of the influencing. Transport Policy, 121-129.

22. Schipper, F. K. (2009). Trends in truck freight energy use and carbon emissions in selected OECD countries from 1973 to 2005. Energy Policy, 3743-3751.

23. SCT, S. d. (11 de Mayo de 2015). Estadistica basica 2014. Recuperado el 24 de Agosto de 2015, de Estadistica basica 2014: http://www.sct.gob.mx

24. Sorrella, S., Lehtonen, M., Stapleton, L., Pujol, J., \& Champion, T. (2009). Decomposing road freight energy use in the United Kingdom. Energy Policy, 31153129. 
25. Sorrella, S., Lehtonen, M., Stapleton, L., Pujol, J., \& Champion, T. (2012). Decoupling of road freight energy use from economic growth in the United Kingdom. Energy Policy, 84-97.

26. Sterman, J. D. (2000). Business Dynamics, Systems Thinking and Modeling for a Complex World. United States: McGraw-Hill Higher Education.

27. Vanek, F., \& Campbell, J. (1999). UK road freight energy use by product: trends and analysis from. Transport Policy, 237-246. 